\title{
The Measurement of Remaining Capacity of the Battery Based on C8051F022
}

\author{
Zuojun Song \\ Automotive Engineering Department, Zibo Vocational Institute, The West \\ End of Liantong Road, Zibo 255314, Shandong Province, P.R. China \\ songzuojun@126.com
}

\begin{abstract}
.
A remaining capacity system based on C8051F022 was introduced. Architecture of the system was described, and hardware circuit was introduced in details, especially anaysed the electronic capacity acquisition circuit, and the design of the software was presented. The system had the fuction of parameter setting, data collection and data display. The system had been manufactured and measured for the sake of simulation. Such as high accuracy and high reliability etc. was shown for the system by practical test.

Keywords: battery; remaining capacity; internal resistance; C8051F022; signal chip.

\section{Introduction}

The battery is a complex electrochemical system, which is widely used in aerospace, communication, electric power, finance, transportation and other fields. The electrical energy to use is different with different load conditions in battery. And the electrical energy to consume is also declined with the increase of use time. The electrical energy of battery affects the systemic reliability. In some important areas, the remaining electrical energy of battery is real-time tested, which ensures the good state of the system, has important practical significance. In view of this situation, this paper has designed a kind of intelligent measuring system with C8051F022 as the core control unit, to realize the measurement of the electrical equipment of the remaining capacity[1,2,3].
\end{abstract}




\section{The principle of internal resistance measuring battery remaining electrical energy $[4,5]$}

The research indicates that there is about 0.88 correlation between the battery internal resistance and capacity, by measuring the internal resistance of the battery, can more accurately predict the remaining battery power.

The internal resistance of battery reduces gradually for charging, and its internal resistance increases gradually for discharging. With the aging of the battery, the resistance increases gradually, and the capacity also decreases. According to the research, when the capacity of the battery is higher than $87 \%$, the internal resistance of the battery is basically unchanged. On the other hand, the internal resistance of the battery slowly increases. When the capacity of the battery is few, the internal resistance of battery will increase rapidly. The internal resistance of the battery is $2 \sim 4$ times that the battery is fully discharged compared with is fully charged.

The characteristic of the battery is analyzed by the method of impedance. The ohm resistance numerical $\mathrm{R}_{\Omega}$ represents charge level of the battery. In order to facilitate the measurement, the pure resistance $\mathrm{R}$ is isolated from the equivalent impedance $\mathrm{Z}$ usually. There is a linear relationship between $\mathrm{R}$ and $\mathrm{R} \Omega$, the degree of the battery charge is expressed indirectly by $\mathrm{R}$.

The internal resistance of the battery is very small, the impedance of measuring wire becomes negligible, so the method of measurement for four wires is used, that the drive current loop is separated from the induction voltage circuit. The constant current of AC audio is applied in the battery between two electrodes, and the terminal voltage of the battery is detected, and the angle between the voltage and the current.

The battery is charged fully, and then it is discharged with the common rate, the internal resistance and the electrical energy is recorded. When the battery is discharged and need to be charged, the complete discharge curve is obtained, that is the relationship curve between the remaining power and resistance. This curve is stored in EPROM, the batteries are measured of the same model and the 
specifications, according to the internal resistance measured online, through checking the table and calculating, the single board computer will obtain the remaining capacity.

\section{The hardware of the system is designed}

The structure diagram that the rest of the electricity measured online is shown in figure 1. It is consisted of audio signal generator, drive coupling circuit, differential amplifier circuit, filter network, rectifies circuit, the circuit that the phase is detected, the sampling circuit of voltage and current, the single chip microcomputer C8051F022, LCD display (LCM12864) and the keyboard, and so on.

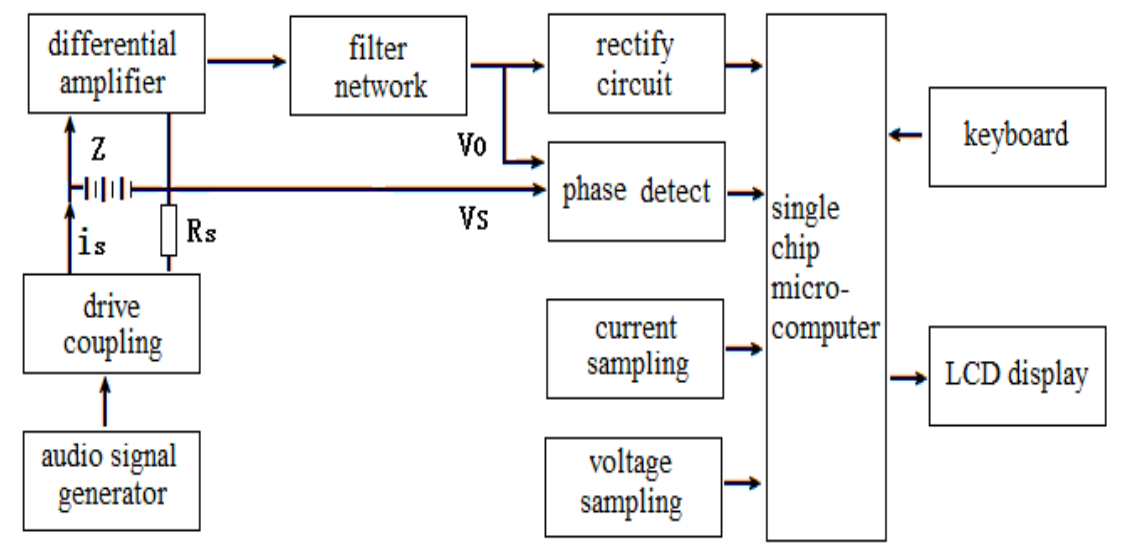

Fig. 1. The hardware block diagram

Except for the electric circuit and the mechanical part, Electric circuit and the mechanical points, using the technology of system on chip, the other circuits of the system are integrated together, it can reduce the power consumption of the system.

\subsection{The interface is designed of the system[5]}

The hardware modules are combined through the software, with a small number of sensors and execution modules in the outside, the online measuring that remaining power of the battery is implemented. The interface connection circuit of the single chip microcomputer and peripheral device is shown in figure 2. 
P0: keyboard input, $\mathrm{P} 0.0 \sim \mathrm{P} 0.5$, connect the keyboard. P1: simulation input, P1.0 P1.3, connect the SPI line corresponding with A/D converter. P2: the data interface of LCD 12864. P3: the control interface of LCD. Among them, P3.5 is Piece of optional signal, P3.6 is read and write signal, P3.7 is energy produced signal.

\subsection{The phase detection circuit[6]}

The phase must be detected real-time exerted to the power, in order to control the voltage supplied to the power. A $6 \mathrm{v}$ voltage is output from the secondary coil of the transformer; it is used for initial phase detection, the low voltage power supply through a full-wave rectifier, voltage to reduce, shaping and amplification, it is output to the input of the single chip microcomputer external interrupt, and completes phase detection.

\subsection{The circuit of voltage is collected[6]}

The acquisition of residual voltage is completed by single-phase and two-way power chip CS5460. CS5460A is a modulus converter of a highly integrated that contains ADC, high-speed calculation function of electric power, a serial interface. It can accurately measure and calculate active electronic energy, instantaneous power, etc. It can measure current with the low cost shunt or transformer, it can measure the voltage with the partial pressure resistance or voltage transformer. The voltage signal is as input signal of the CS5460 after operation and amplifier in the instrument, the circuit principle diagram is shown in figure 3. 


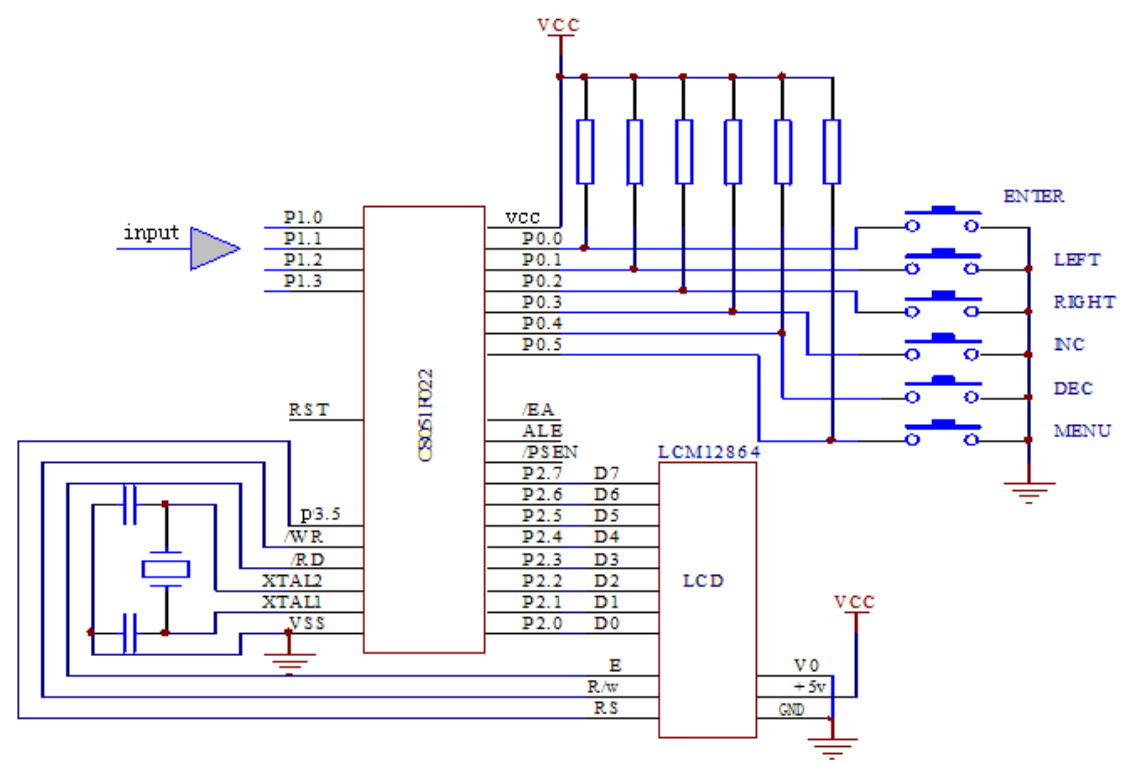

Fig. 2. Peripheral interface circuit diagram of the single chip microcomputer $+5 \mathrm{v}$ L1

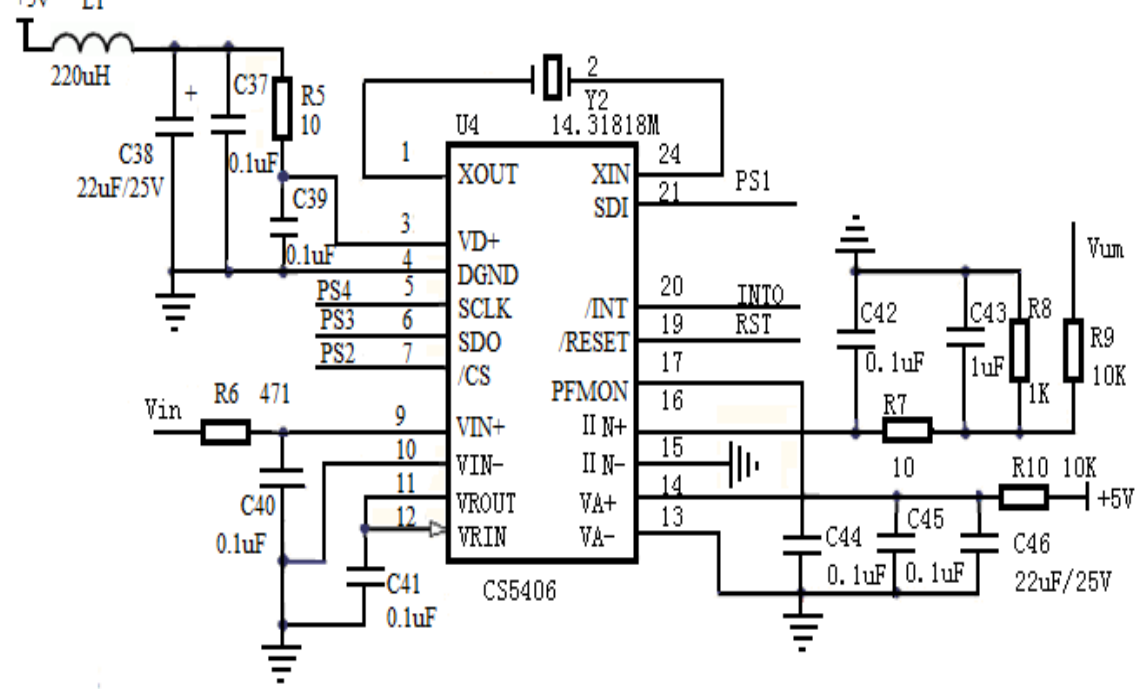

Fig. 3. The voltage acquisition circuit

\subsection{The key circuit}

There are 6 control buttons in the system, corresponding $6 \mathrm{I} / \mathrm{O}$ interfaces of the C8051F022. The interface is received $+3.3 \mathrm{~V}$ voltage through pull-up resistor, and 
always keeps the high level, when the button is pressed, grounded directly, triggered into a low level.

\subsection{Liquid crystal display circuit}

LCM12864 is mainly composed of the LCD panel array driver circuit KS0108B, the matrix display controller KS0107B, LCD display and LED backlight, thus form a complete display system module. The controller is the core of the whole display system, it has own clock signal, and provide a complete set of instruction system. Among them, two pieces of KS0108B controls two parts of the display respectively. The LCD connects to the CPU through the bus, realizing the read and write operations to the external data, and also changes the position of the display window easily.П II

\section{The software of the system is designed[5]}

The modular programming is used to software design, it contains the main program module and subroutine module. The subroutine is made up of the A/D interrupt handling subroutine and the timer interrupt subroutine. The main task of the main program is to complete the initialization, calculate the cycle $\mathrm{T} 2$ of the square wave, control the opening and closing of the interruption, complete the judgment of the start and end, and has the function that calls to each subroutine.

The timer/counter of standard 80C51 single chip microcomputer can measure pulse width, its working principle is shown as follows. It uses INTO as control port of timer/counter T0, when TR0 and GATE is set to 1 , the INTO is only high level, the timer/counter T0 strobes to work and counts the clock pulse. Due to the $\mathrm{T} 2$ is determined by the Rset, changed for a little in the process of detection, this feature can be used to simplify the process of sampling, improve the duty ratio calculation speed.

The system adopts digital filtering method. The extremums are deleted and using average filter. The data is sampled continuous, get rid of the maximum and 
minimum values, and then get the average of the remaining data. According to the collected data and chooses the appropriate range, so that its precision is higher.

The input signal of the keyboard is recognized in order to test the battery, get $\mathrm{V} 0$ and Vs, calculate the corresponding $\mathrm{Z}$ values, thus calculates the battery internal resistance R. According to the relationship that the remaining power stored in the EPROM and the resistance, the voltage of the battery is determined that the internal resistance $\mathrm{R}$ has get. The communication is formed between human and machine through the data showed.

\section{Test results}

A DLT-B8500 instrument is used to measure the battery that has a 2v3000ah VRLA, and compares the measurement method proposed in this paper. The sinusoidal ac signal is adopted to measure, the amplitude is $100 \mathrm{mV}$, resistance $\mathrm{R}$ is $0.5 \Omega$, isolated dc capacitance $\mathrm{C}$ is $3300 \mu \mathrm{F}$. Specific results are shown in table 1. Setting the temperature transmission ratio, heating the temperature sensor with a hair dryer, checking the temperature changes on the display.

Table 1. the test data of the battery internal resistance

\begin{tabular}{c|c|c}
\hline $\begin{array}{c}\text { serial } \\
\text { number }\end{array}$ & $\begin{array}{c}\text { instrument } \\
\text { measurements }(\Omega)\end{array}$ & $\begin{array}{c}\text { this paper measured } \\
\text { value }(\Omega)\end{array}$ \\
\hline 1 & 0.059 & 0.052 \\
\hline 2 & 0.062 & 0.060 \\
\hline 3 & 0.060 & 0.056 \\
\hline 4 & 0.058 & 0.053 \\
\hline
\end{tabular}

\section{Conclusions}

In this paper, a measurement system of the remaining power based on C8051F022 microprocessor is presented, the working principle and control mode was described in details, finally the feasibility of the measurement system is verified further through the experiment. As long as the amplitude of the current source is changed, the capacity of the various type batteries can be measured using 
the resistance method. This method has a lot of characteristics, such as high precision, simple operation, strong practicality and generality, etc.

\section{References}

[1]. H.Y. Fu. Study and Implementation of Estimation Algorithm on SOC for Electric Vehicle Battery, D. Hangzhou Dianzi University. Hangzhou, Zhejiang Province, P.R. China(2010).

[2]. F.P. Li. Research on Internal Resistance Measurement for Health Diagnosis of the Lead-Acid Battery, D. Nanjing University of Aeronautics and Astronautics. Nanjing, Jiangsu Province, P.R. China(2009).

[3]. Y. Chen, C.F. Song. Design of Temperature Control System Based on C8051F040, J. Industrial Control Computer, Vo27. 59-60(2014).

[4]. Y.S. Feng, J.J. Song. The Design of Temperature and Humidity Measurement and Control System Based on Single-chip Microcomputer, J. Journal of Shandong University of Technology(Natural Science Edition, Vo28. 19-23(2014).

[5]. F. Li. The Measurement of Remaining Capacity of Storage Battery Based on SOC Technology C8051F02, J. Power Supply Technologies and Applications, Vol.13-16(2010).

[6]. Y.L. Fu, H.Q. Xiao, H.W. Wang, X.X. Li. Design of the Residual Voltage System Based on C8051F021, J. Electronic Test, Vol.11-14(2012). 